\title{
artigo
}

Ziani, J.S.; Balk, R.S.; Gomes, N.S.; Rodrigues, M.P.; Figueredo, B.G.; Cardozo da Silva, B.

A influência de um Programa de Educação Tutorial em Saúde Coletiva na formação acadêmica

DOl: https://doi.org/10.36489/saudecoletiva.2021v11i67p6829-6838

\section{A influência de um Programa de Educação Tutorial em Saúde Coletiva na formação acadêmica}

The influence of a tutorial education program in public health on academic training La influencia de um programa de enseñanza tutorial em salud pública em la formación académica

\begin{abstract}
RESUMO
Objetiva-se relatar a magnitude das experiências vivenciadas por acadêmicos egressos da equipe do programa de educação tutorial em saúde coletiva para a formação acadêmica. Trata-se de um relato de experiência a partir de informações advindas de acadêmicos egressos do PET PISC. A qual tornou-se possivel identificar que o programa cumpriu de forma fidedigna com o seu papel ao longo desses 10 anos, uma vez que ele conseguiu formar acadêmicos com o entendimento sobre os aspectos da interdisciplinaridade e integralidade, profissionais com destaque no mercado de trabalho, e com conhecimento sobre as reais noções da saúde pública, além ter contribuído de forma efetiva para a inserção de inúmeros acadêmicos nos programas de pós-graduação. Por fim, percebe-se que o programa atingiu o seu propósito de formar profissionais qualificados, críticos, reflexivos, socialmente comprometidos e autônomos, garantindo aos acadêmicos egressos entrevistados neste estudo, um diferencial no mercado de trabalho. DESCRITORES: Relações Interprofissionais; Saúde coletiva; Desempenho profissional.
\end{abstract}

\section{ABSTRACT}

The objective is to report the magnitude of the experiences lived by academics graduating from the team of the tutorial education program in collective health for academic training. This is an experience report based on information from academics who graduated from PET PISC. Which became possible to identify that the program reliably fulfilled its role over these 10 years, since it was able to train academics with the understanding of aspects of interdisciplinarity and integrality, professionals especially in the labor market, and with knowledge about the real notions of public health, in addition to having effectively contributed to the insertion of numerous academics in graduate programs. Finally, it is clear that the program has achieved its purpose of training qualified, critical, reflective, socially committed and autonomous professionals, ensuring the graduates interviewed in this study a differential in the labor market. DESCRIPTORS: Interprofessional Relations; Public Health; Work Performance.

\section{RESUMEN}

El objetivo es informar de la magnitud de las experiencias vividas por los académicos que se graduan del equipo del programa de educación tutorial en salud colectiva para la formación académica. Se trata de un informe de experiencia basado en la información de los académicos que se graduaron de PET PISC. Que se hizo posible identificar que el programa cumplió de manera fiable su función durante estos 10 años, ya que fue capaz de formar académicos con la comprensión de aspectos de interdisciplinariedad e integralidad, profesionales especialmente en el mercado laboral, y con conocimiento sobre las nociones reales de salud pública, además de haber contribuido efectivamente a la inserción de numerosos académicos en programas de posgrado. Por último, está claro que el programa ha logrado su propósito de formar profesionales cualificados, críticos, reflexivos, socialmente comprometidos y autónomos, asegurando a los graduados entrevistados en este estudio una diferencia en el mercado laboral. DESCRIPTORES: Relaciones interprofesionales; Salud Pública; Rendimiento Laboral.

RECEBIDO EM: 27/02/2021 APROVADO EM: 08/03/2021

\section{Jarbas da Silva Ziani}

Acadêmico de Enfermagem, pela Universidade Federal do Pampa - UNIPAMPA.

ORCID: 0000-0002-9325-9390

\section{Rodrigo de Souza Balk}

Doutor em ciências biológicas, professor pela Universidade Federal do Pampa - UNIPAMPA.

ORCID: 0000-0001-5254-6732 


\section{Natália da Silva Gomes}

Enfermeira, Residente em Atenção Básica, pela Universidade do Vale do Rio do Sinos.

ORCID: 0000-0002-6952-7172

\section{Marília Pacheco Rodrigues}

Acadêmica de Enfermagem, pela Universidade Federal do Pampa - UNIPAMPA. ORCID: 0000-0001-5140-5516

\section{Bárbara Garcia Figueredo}

Acadêmica de Fisioterapia, pela Universidade Federal do Pampa - UNIPAMPA. ORCID: 0000-0002-8577-0906

\section{Bruna Cardozo da Silva}

Acadêmica de Enfermagem, pela Universidade Federal do Pampa - UNIPAMPA. ORCID: 0000-0002-5181-4763

\section{INTRODUÇÃO}

A garantia do direito à saúde e à educação foi uma conquista de toda a sociedade brasileira e esteve diretamente associada à construção do Estado democrático de direito, resultado de um grande pacto social, expresso por meio da Constituição Federal de $1988^{1}$. Sob esse aspecto, foi criado o Sistema Único de Saúde (SUS) um dos maiores e mais complexos sistemas de saúde pública, a qual os princípios e diretrizes estão ancorados na equidade, universalidade, integralidade, descentralização e participação social ${ }^{2}$.

Visando o atendimento integralizado previsto no SUS, os cursos de graduação na área da saúde devem buscar formar profissionais capazes de atender às reais necessidades da população ${ }^{2}$. Atrelado a isso, em 1979 criou-se o programa especial de treinamento, a qual atualmente é cognominado Programa de Educação Tutorial (PET). O Programa foi desenvolvido por meio de uma estratégia do Ministério da Educação (MEC) na prorrogativa de estimular a educação tutorial e a indissociabilidade do tripé ensino, pesquisa e extensão nas Instituições de Ensino Superior (IES) do país, através da composição de grupos de acadêmicos ligados a um tutor ${ }^{3}$.

Similarmente, por volta dos nos anos 70, surge em consonância com a Reforma Sanitária o campo da Saúde Coletiva (SC), que vem para integrar a perspectiva de constituir um paradigma que permitisse uma nova articulação entre as diferentes instituições do campo da saúde ${ }^{4}$. Estabelecendo-se por meio de uma crítica sistemática do universalismo naturalista do modelo biomédico com à amplitude dos aspectos sociais envolvidos no processo saúde, adoecimento e cuidado5.

À vista disso, verifica-se que a SC interage diretamente com os preceitos do PET, uma vez que ele também visa a interação entre os acadêmicos e sociedade de forma ativa, atuando efetivamente na melhoria da saúde dos indivíduos, por meio de um olhar multidimensional onde se leve em consideração aspectos como, a família, comunidade, cultura, estrutura social e desenvolvimento físico 5 .

Então, surge o Programa de Educação Tutorial Práticas Integradas em Saúde Coletiva (PET PISC), o qual tem por objetivo integrar acadêmicos dos cursos de graduação em Enfermagem, Farmácia e Fisioterapia de forma integrada e interdisciplinar, na prestação dos cuidados domiciliares junto às comunidades do município em que estão inseridos para a promoção da saúde, a reabilitação de danos, agravos e prevenção de doenças, visando a formação em saúde coletiva, o qual propõem-se desenvolver competências para o cuidado individual e coletivo, resultando na qualificação de profissionais críticos e comprometidos com a melhoria da saúde e com sua performance na equipe ${ }^{6,7}$.

Além do mais, vale realçar o compromisso que o programa PET detém na formação dos acadêmicos, uma vez que ele propõe-se promover vivências aos acadê- micos que os levem a desenvolver valores, como, habilidade de trabalhar em grupo, amadurecimento, organização, autonomia, autoconfiança, conhecimento técnico-científico e habilidade em pesquisa ${ }^{3}$. Corroborando com essa premissa, sabe-se que o programa possui uma formação diferenciada, pela relação mais próxima que possui entre serviços públicos de saúde e a universidades, também por instigar a discussão constante de propostas curriculares que se articulem de forma eficaz no previsto nas Diretrizes Curriculares Nacionais dos cursos de graduação da área da saúde ${ }^{8}$.

Assim, o presente estudo tem como propósito relatar a importância das experiências vivenciadas pela equipe do programa PET PISC na formação de futuros profissionais da área da saúde.

\section{MÉTODO}

Trata-se de um relato de experiência a partir de informações advindas de acadêmicos egressos do PET PISC. O estudo foi desenvolvido no período de maio a julho de 2020, com a participação de acadêmicos egressos do grupo PET PISC pertencentes aos cursos de graduação em Enfermagem, Farmácia e Fisioterapia os quais tivessem uma vivência mínima de 6 meses no programa. Foram excluídos do estudo os egressos que durante suas participações no referido programa não realizaram alguma das atividades da tríade ensino, pesquisa e/ou extensão, que não responderam a alguma pergunta do questionário e que 
tiveram sua formação além do tempo previsto, uma vez que o PET prima pela excelência na formação acadêmica.

O Programa extensionista teve suas atividades iniciadas em 2010 durante as práticas do componente curricular de graduação denominado Saúde Coletiva do curso de graduação em Enfermagem. Atualmente o PET conta com a participação dos cursos de graduação em Enfermagem, Farmácia e Fisioterapia da Universidade Federal do Pampa, campus Uruguaiana, Rio Grande do Sul.

Ao longo de seus 10 anos, o Programa expandiu suas ações para diversos setores da Atenção Primária à Saúde (APS) do município, tais como, escolas da rede municipal e estadual, Estratégias de Saúde da Família (ESF), Centro de Atenção Psicossocial (CAPS), Prefeitura Municipal de Uruguaiana, Programas de Pós Graduação, Associação de Apoio a Pessoas com Câncer (AAPECAN), Centro de Referência Especializado de Assistência Social (CREAS), assim como, o lar da velhice São Vicente de Paulo.

Tendo em vista a abordagem interdisciplinar do grupo, as visitas domiciliares (VD) ocorrem semanalmente compostas por acadêmicos dos cursos que compõem o PISC. Além do mais, frisa-se que o objetivo das VD's desenvolvidas pelo grupo viabiliza o acompanhamento das famílias e dos indivíduos de forma integral, através de um olhar interdisciplinar, com a integração dos agentes comunitários em saúde e equipe de enfermagem do território adstrito. fortalecendo assim, o vínculo entre a equipe e os acadêmicos proporcionando a troca de conhecimento e experiência.

Já no que tange às atividades de ensino, o grupo realiza ações nas escolas a fim de levar informação para as crianças na forma de filmes, animações ou desenhos infantis, com a atividade intitulada "Tela PISC", também desenvolve feiras de promoção à saúde a toda a população do município, como campanhas de conscientização, ações de primeiros socorros, campanhas alusivas à saúde da criança, mulher, homem através de materiais didáticos que são distribuídos para a comunidade.
$\mathrm{Na}$ pesquisa o programa busca instigar os acadêmicos a prática da escrita e da oralidade, sendo fortalecidas através da leitura e apresentação de artigos além de participação em congressos voltados para a área da saúde coletiva. A vista disso, as pesquisas incentivam a participação e $\mathrm{o}$ desenvolvimento de trabalhos científicos, aprimorando o conhecimento do acadêmico e instigando um dos objetivos do PET que é o ingresso em programas de pós graduação, além de crescimento profissional, as pesquisas estimulam também o crescimento pessoal, aperfeiçoando a visão crítica e a persistência.

Dessa forma, pensando sempre na aproximação e no aprimoramento do pro-

\section{Na pesquisa}

o programa

busca instigar

os acadêmicos a

prática da escrita

e da oralidade,

sendo fortalecidas

através da leitura

e apresentação de

artigos além de

participação em

congressos voltados

para a área da saúde

coletiva. grama decidiu-se estruturar e disponibilizar um formulário nas páginas oficiais do grupo PET PISC, sendo elas: Instagram, Facebook e site da instituição para que os acadêmicos egressos pudessem relatar suas experiências vivenciadas no Programa.

O formulário dispunha de questionamentos sobre as vividas pelos acadêmicos no tempo em que integrou o PET PISC, tais como: inserção no mercado de trabalho, pós graduação, e as contribuições do Programa para a formação acadêmica. As questóes fechadas foram analisadas através de uma análise descritiva dos dados e as questões abertas foram interpretadas com muito cuidado para seguir fidedignamente a opinião do participante. Além do mais, que se cumpriu todos os aspectos éticos a fim de garantir o anonimato dos participantes os mesmos nesse manuscrito foram identificados como P1 e P2.

\section{RESULTADO E DISCUSSÃO}

Participaram desta atividade 26 acadêmicos egressos, sendo 23 do sexo feminino e 3 masculinos. Desses, 13 possuem graduação em Enfermagem, 9 Fisioterapia, 4 Farmácia. Assim, constatou-se que a inserção dos acadêmicos no PET PISC promoveu a compreensão entre os saberes de forma integrada e interdisciplinar, uma vez que durante a graduação os acadêmicos relatam não trabalharem de forma integra$\mathrm{da}$, visto que os componentes curriculares dos três cursos possuem uma matriz curricular muito segregada por áreas, viabilizando em uma carência do conhecimento na sua forma integral e principalmente no que tange a saúde coletiva. Todavia, o curso de Enfermagem destacou-se como um dos que possui essa área mais consolidada na sua formação.

Assim, o Programa trabalha no intuito de suprir esse aspecto através do trabalho interdisciplinar onde há o compartilhamento de saberes entre as diversas áreas do conhecimento, seja pelas trocas entre os acadêmicos, seja do tutor, sempre enfocando para aproximar os conteúdos teóricos desenvolvidos nos componentes curriculares com as vivências práticas no Programa, 
por intermédio de suas atividades extensionistas, assim, instigando do acadêmico um pensamento crítico e reflexivo?.

Considerando esse contexto, a estratégia empregada pelo PET PISC traz resultado semelhante a um estudo, a qual desenvolveu com os acadêmicos a criação de um estilo de pensamento interdisciplinar e interprofissional na saúde, e os resultados mostram que para ser consistente é necessário que ocorra a ampliação das experiências educacionais e de trabalho ${ }^{10}$. Sendo assim, é necessário a interação entre diferentes coletivos de pensamentos e conhecimentos, para ocorrer a produção de novos significados sobre o cuidado à saúde, de modo orientado às necessidades do usuário ${ }^{11}$. Fatos esses que podem ser evidenciados pelas falas dos acadêmicos infracitados.

"O trabalho multiprofissional com certeza foi a maior contribuição. Além das vivências do SUS, hoje atuo em um hospital do SUS e vejo como o conhecimento anterior me ajudou." (P1).

"Uma maior abordagem e conhecimentos em todas as áreas da saúde pública, desde a urgência e emergência até a área da atenção básica." (P2).

Identificou-se também que o sentimento de gratidão se fez muito presente nas respostas dos acadêmicos egressos, pelo Programa ter influenciado imensamente na sua compreensão sobre o trabalho em equipe, trazendo benefícios a sua vida profissional. O trabalho em equipe é um dos pilares do programa PET PISC, uma vez que se acredita que é por meio dele que os acadêmicos poderão vir a desenvolver competência mínimas a serem atingida dentro do seu processo formativo.

Ademais, observa-se, que a perspectiva interdisciplinar que é desenvolvida no programa contribui consideravelmente para a valorização do trabalho em equipe no enfrentamento dos desafios do grupo em campo de prática, pois ele é um dos componentes estratégicos de enfrenta- mento das crescentes complexidades, tanto das necessidades de saúde que requerem uma abordagem ampliada e contextualizada como da organização dos serviços e dos sistemas de atenção à saúde em rede ${ }^{12}$.

\section{O trabalho em}

equipe é um dos

pilares do programa

PET PISC, uma vez

que se acredita que é

por meio dele que os

acadêmicos poderão

vir a desenvolver

competência

mínimas a serem

atingida dentro

do seu processo

formativo.

Nesse sentido, ressalta-se que o grupo só consegue atingir esses objetivos, pois ele vem rompendo com o modelo Flexneriano, a qual ainda é muito empregado em várias Universidades, em que predomina a pouca conexão interprofissional e falta de trabalho em equipe ${ }^{13}$. Assim, no PET PISC, os estudantes são fomentados a trabalhar o oposto desse modelo tendo interação entre os membros e tarefas em conjunto. Uma vez que promovem entendimento da importância do engajamento de diversos profissionais da saúde, cada um contribuindo de forma dinâmica com os conhecimentos específicos de sua área de formação e assim, alcançando o objetivo que é desenvolver a comunicação efetiva do acadêmico e a equipe de trabalho, reconhecimento do trabalho nos demais componentes da equipe, interdependência para a realização das ações, colaboração interprofissional e a atenção centrada no usuário ${ }^{12}$.

Quanto ao indicador sobre o ingresso dos acadêmicos no mercado de trabalho, pode inferir que ele foi satisfatório, uma vez que $88 \%$ (23) dos acadêmicos egressos obtiveram sucesso na sua inserção ao mercado de trabalho em menos de seis meses de formado. Dado esse que reflete de outro estudo, a qual $55 \%$ conseguiram adentrar ao mercado de trabalho em menos de seis meses, dessa forma podendo inferir que o PET contribui significativamente para a criação de acadêmicos com destaque no mercado de trabalho, uma vez que o Programa objetiva formar profissionais diferenciados capazes de desenvolver-se profissionalmente, quanto pessoalmente e intelectual ${ }^{14}$.

Também, cabe destacar o espírito de liderança que o grupo promove aos acadêmicos, por meio da comunicação, administração e gerenciamento dos problemas enfrentados nos serviços de saúde ${ }^{14}$. Para mais, essa liderança foi referida pelos acadêmicos como um ponto que mais o promoveu destaque em campo prático, seja no comando da sua equipe, seja nas condutas tomadas frente ao paciente.

Quando inspecionado na literatura científica sobra o conceito de liderança pode-se denotar que ela está intimamente ligada com o fator de conquista profissional, visto que infere na produtividade, qualidade e relevância da força de trabalho do profissional ${ }^{15}$. Para mais, estudos corroboram para a necessidade no aumento do número de profissionais com o caráter de liderança arraigado em sua personalidade, pois é o profissional que o mercado de trabalho vem exigindo e encontra-se em menor quantidade-

Similarmente aos aspectos supracitados, evidenciou-se também o comprome- 


\section{artigo}

timento do programa quanto a produção científica, visto que mais da metade dos acadêmicos egressos do programa direcionaram-se para os programas de pós graduação, uma vez que todos desses acadêmicos saíram com publicações desenvolvidas pelo grupo. Além do mais, o grupo clama-se de suma importância explorar os problemas da comunidade trazendo resoluções para o incentivo a melhoria da qualidade de vida da população por meio de suas pesquisas e publicações.

\section{CONCLUSÃO}

Em linhas gerais, percebe-se que o PET PISC atingiu o seu propósito de formar profissionais qualificados, críticos, reflexivos, socialmente comprometidos e autônomos, garantindo aos acadêmicos egressos entrevistados neste estudo, um diferencial no mercado de trabalho. Para mais, ressalta-se que os achados desse estudo discorrem a uma situação de um grupo PET específico e não possui o objetivo de generalizar os achados deste manuscrito, porém vem para identificar os benefícios do programa ao longo desses 10 anos de sua inserção.

Além do mais, cabe destacar a importância de o programa promover a atividades prática juntamente com a graduação, uma vez que oportuniza ao acadêmico vivenciar experiências que não estão presentes em sua matriz curricular, e assim, promovendo uma formação global tornando esses futuros profissionais apitos para integrar uma equipe e ganhar destaque no mercado de trabalho e também oportunizando a inserção dos mesmos nos cursos de pós-graduação. -

\section{REFERÊNCIAS}

1. Silva MCN, Machado MH. Sistema de Saúde e Trabalho: desafios para a Enfermagem no Brasil. Ciênc. saúde coletiva [Internet]. 2020 [acesso em 10 jan 2020]; 25(1): 7-13. Disponível em: http://dx.doi.org/10.1590/1413-81232020251.27572019.

2. Menezes APR; Moretti B, Reis AAC. O futuro do SUS: impactos das reformas neoliberais na saúde pública - austeridade versus universalidade. Saúde em Debate [Internet]. 2019 [acesso em 14 out 2020]; 43(spe5): 58-70. Disponivel em: https://doi. org/10.1590/0103-11042019S505.

3. Torina HF, Almeida LO, de Paula JS. História do Programa de Educação Tutoria da FMRP-USP. Medicina (Ribeirão Preto) [Internet]. 2016 [acesso em: 10 jan 2020]; 49(4): 374-380. Disponivel em: http://www.revistas.usp.br/rmrp/article/view/122731

4. Ferreira J, Brandão ER. Desafios da formação antropológica de profissionais de Saúde: uma experiência de ensino na pós-graduação em Saúde Coletiva. Interface (Botucatu) [Internet]. 2019 [acesso em: 19 out 2020]; 23(e170686): 1807-5762. Disponível em: https://doi.org/10.1590/Interface.170686.

5. de Souza KMJ, Seixas CT, David HMSL, da Costa AQ. Contribuições da Saúde Coletiva para o trabalho de enfermeiros. Rev. bras. enferm. [Internet]. 2017 [acesso em: $24 \mathrm{dez}$ 2020]; 70(3): 543-549. Disponivel em: http://dx.doi.org/10.1590/0034-7167-2016-0401

6. Vieira LM, Sgavioli CAPP, Simionato EMRS, Inoue ESY, Heubel MTC, Contil MHS, et al. Formação profissional e integração com a rede básica de saúde. Trab. educ. saúde [Internet]. 2016 [acesso em 24 dez 2020]: 14(1): 293-304. Disponivel em: http://dx.doi. org/10.1590/1981-7746-sip00093.

7. Duarte M de L, Torres O, Santini N, Balk R. Práticas integradas em saúde coletiva: a experiência de um programa de extensão no sul do país. RCS [Internet]. 2013 [acesso em: 12 ago 2020]; 12(22): 15-9. Disponível em: https://www.revistas.unijui.edu.br/ index.php/contextoesaude/article/view/256

8. Farias SBCS, Noro LRA. PET-Saúde como indutor da formação profissional para o Sistema Único de Saúde. Ciênc. saúde coletiva [Internet]. 2017 [citado em: 22 dez 2020]; 22(3): 997-1004. Disponivel em: https://doi.org/10.1590/141381232017223.15822016
9. Pereira MLAS, Pereira MHQ, Teles BKA, Carvalho RBC, Oliveira ERA de. Interdisciplinaridade em Saúde Coletiva: construção de um recurso didático no campo da prática profissional. Rev. Bra. Edu. Saúde [Internet]. 2019 [citado em: 26 dez 2020]; 9(4): 7783. Disponivel em: https://www.gvaa.com.br/revista/index.php/ REBES/article/view/6935

10. Lima VV, Ribeiro ECO, Padilha RQ, Mourthé JCA. Desafios na educação de profissionais de Saúde: uma abordagem interdisciplinar e interprofissional. Interface (Botucatu) [Internet]. 2018 [citado em: 25 dez 2020]; 22(Suppl 2): 1549-1562. Disponível em: https://doi.org/10.1590/1807-57622017.0722

11. Peduzzi M, Agreli HLF, Silva JAM da, Souza HS. Trabalho em equipe: uma revisão ao conceito e a seus descobrimentos no trabalho interprofissional. Trab. educ. saúde [Internet]. 2020 [acesso em 26 dez 2020]; 18 (supl.1): Disponivel em: http://dx.doi. org/10.1590/1981-7746-sol00246.

12. Scherer MD dos A, Marino SRA, Ramos FRS. Rupturas e resoluções no modelo de atenção à saúde: reflexões sobre a estratégia saúde da família com base nas categorias kuhnianas. Interface (Botucatu) [Internet]. 2005 [citado em: 14 oct 2020]; 9(16): 53-66. Disponivel em: http://dx.doi.org/10.1590/S141432832005000100005.

13. Lopes TF, Silva BV da S, de Carvalho LS, Vaz SS, Pereira JM, de Carvalho REF de L. Atuação profissional dos egressos do programa de educação tutorial de um curso de enfermagem. Revista Enfermagem Contemporânea [Internet]. 2020 [acesso em: 26 dez 2020]; 9(2): 211-217. Disponível em: http://dx.doi. org/10.17267/2317-3378rec.v9i2.2913.

14. Neves VR, Sanna MC. Conceitos e práticas de ensino e exercício da liderança em Enfermagem. Rev. Bras. Enferm. [Internet]. 2016 [citado em: 24 nov 2020]; 69(4): 733-740. Disponivel em: https://doi.org/10.1590/0034-7167.2016690417i.

15. Mendes IAC, Ventura CAA, Trevizan MA, Marchi-Alves LM, Souza JVD de. Education, leadership and partnerships: nursing potential for Universal Health Coverage. Rev. Latino-Am. Enfermagem [Internet]. 2016 [citado em: 26 dez 2020]; 24: e2673. Disponivel em: https://doi.org/10.1590/1518-8345.1092.2673. 